\title{
Glare sensitivity and visual acuity after excimer laser photorefractive keratectomy for myopia
}

\author{
Ulrike Niesen, Urs Businger, Peter Hartmann, Peter Senn, Isaak Schipper
}

\begin{abstract}
Background-Following excimer laser photorefractive keratectomy (PRK), an increase in glare sensitivity and a reduction in contrast sensitivity can occur owing to changes in the cornea (structure and topography). In this study, an attempt was made to quantify and document objectively a change in those subjective perceptual factors.

Methods-Snellen visual acuity and disability glare were measured with the Berkeley glare test preoperatively as well as $1,3,6,9$, and 12 months postoperatively, after excimer laser photorefractive keratectomy (PRK) on 32 myopic patients (46 eyes). During the postoperative progress checks, haze was graded and contrast sensitivity was measured with the Vistech chart. All the data were statistically analysed by multiple regression.

Results-One year after PRK, a reduction in visual acuity (VA) measured with the low acuity contrast chart $(10 \%)$ with and without glare could still be found, despite the fact that acuity measurements with a high contrast Snellen chart showed the same VA 6 months postoperatively as well as before the treatment. The lowest VA could be measured 1 month postoperatively; thereafter, the acuity increased despite the increase in haze that occurred during the first 3 months.

Conclusion-Disability glare and a reduction in contrast sensitivity could be observed in most patients after PRK treatment with the Meditec laser system with its scanning slit. The future will show if new technology and a broader flattening area of 6 to $7 \mathrm{~mm}$ can minimise these postoperative complications.

(Br f Ophthalmol 1997;81:136-140)
\end{abstract}

Eye Clinic, Cantonal Hospital, Lucerne, Switzerland U Niesen

U Businger

P Hartmann

P Senn

I Schipper

Correspondence to: I Schipper, MD, Cantonal Hospital, 6000 Lucerne 16, Switzerland

Accepted for publication 14 November 1996
The first publications concerning photorefractive keratectomy (PRK) for the treatment of myopia already pointed to the complication of losing optimal visual acuity, particularly a reduction of the VA in conjunction with glare. ${ }^{12}$ Stray light, as caused by the structural changes in the stroma known as haze, has been discussed as a possible reason for the increased glare sensitivity after PRK. ${ }^{34}$

Glare sensitivity can be measured with various instruments. ${ }^{5-14}$ The Berkeley glare test ${ }^{15}$ is a new instrument which fulfils all the criteria set by the American Academy of Ophthalmology. ${ }^{16}$ Elliott and Bullimore have demonstrated that the test yields reliable results and can be easily administered. ${ }^{17}$

The objective of this study was to determine if VA changes among myopes under the condition of glare could be quantified postoperatively. The relation between glare, Snellen acuity, haze, and contrast sensitivity was also analysed.

Patients and methods

For all procedures, an argon fluoride excimer laser MEL 60 (Meditec Aesculap, Heroldsberg, Germany), with a wavelength of $193 \mathrm{~nm}$, operating in a scanning slit mode, was used. The details of the surgical procedure have been described previously. ${ }^{18}$ The eye was fixated by vacuum with the help of a suction ring. The mask contains an iris diaphragm and a suction device to remove debris during the treatment. The fluence at the cornea is $250 \mathrm{~mJ} / \mathrm{cm}^{2}$, with a pulse duration of $20 \mathrm{~ns}$, and a scanning frequency of $20 \mathrm{~Hz}$. The ablation rate is $0.9 \mu \mathrm{m}$ per scan. The diameter of the diaphragm was 5 $\mathrm{mm}$ without the tapered transition zone (TTZ) and $6-7 \mathrm{~mm}$ with the peripheral junction of $1-2 \mathrm{~mm}$ of the TTZ.

\section{PATIENTS}

A total of 32 myopic patients (46 eyes), with a mean age of 37 (SD 9) years (range 26-57) were treated with PRK. Only patients tested with the Berkeley glare test preoperatively and postoperatively (up to 1 year) were included in the analysis. The mean spherical equivalent of the preoperative refraction was -7.7 (SD 2.9) $\mathrm{D}(-2.75$ to $-13.63 \mathrm{D})$. The astigmatism measured between 0 and $4 \mathrm{D}$. The mean ablation was 6.5 (SD 2.0) D (3.0-9.0 D). For the purposes of the evaluation, the patients were divided into three groups according to their preoperative refraction.

Group $I-2.75$ to $-6.0 \mathrm{D}$, mean -4.01 (SD $0.84) \mathrm{D}$ (10 patients/13 eyes)

Group II -6.25 to $-10.0 \mathrm{D}$, mean -7.75 (SD 1.10) D (15 patients/23 eyes)

Group III -10.25 to -14.0 D, mean -11.49 (SD 1.36 ) D (7 patients/10 eyes)

For all refractive errors above $-10.0 \mathrm{D}$, the surgery was aimed at a significant reduction in myopia, but not for emmetropia. All patients were exhaustively informed about the procedure and the risks involved. Local anaesthesia was achieved with oxybuprocaine hydrochloride (Novesin $0.4 \%$ ). To facilitate the removal of the epithelium, cocaine drops $(2 \%)$ were applied preoperatively. Immediately after surgery, a soft contact lens (New-Vues, Ciba 


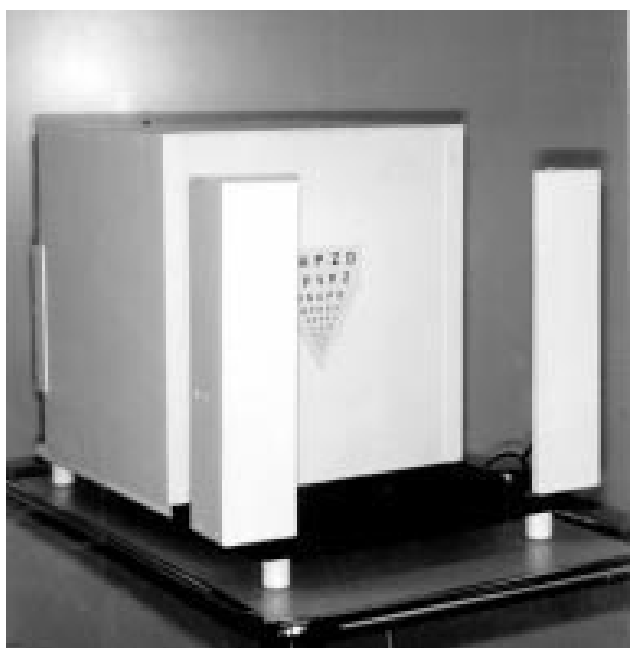

Figure 1 Berkeley glare tester

Vision) was fitted and worn for an average of 3 days (2-4 days) until the erosion had completely healed. Medication during that time consisted of the antibiotics neomycin and polymyxin $(3 \times 1)$ (Neosporin) and a nonsteroidal antiphlogistic diclofenac $(4 \times 1)$ (Voltaren ophthal). After epithelial healing, $0.1 \%$ clobetasone steroid drops (Cloptison) were used, and the dosage tapered off between 3 to 6 months; dosages depended on the degree of myopia and the regression. On average, the dosage was $5 \times 1$ for the first month, $4 \times 1$ for the second, and $3 \times 1$ for the third month. A detailed examination including refraction, slit-lamp, tonometry (Goldmann), pachymetry and biometry, corneal topography (TMS), glare (Berkeley), and contrast sensitivity (Vistech 6500) was performed pre- and postoperatively during the entire observation period of 1 year. All measurements were made by an independent person and not the surgeon concerned.

The Snellen acuity was recorded as the last line in which the patient could correctly identify at least four out of five presented letters.

The Berkeley glare test was used to measure glare sensitivity (Fig 1). It consists of two Bailey-Lovie test charts with different contrasts. ${ }^{19}$ The test charts had to be identified under varying illumination conditions. At the commencement of the test, the letters on the high contrast charts ( $96 \%$ contrast) had to be correctly identified. The VA measured under such conditions should correlate with the Snellen VA. The VA is then measured with the low contrast ( $10 \%$ contrast according to Michelson) charts under four different glare conditions.

Each correctly identified letter was counted and the final result expressed as a number called the visual acuity rating (VAR). Decimal notation is also used to facilitate comparison with other measurements (Table 1).

The haze was evaluated by slit-lamp observation and graded according to the scale of Gartry and Fantes ${ }^{20}$ (scale 0-4, $0=$ clear cornea, $4=$ dense scarring).

Contrast sensitivity was measured by the Vistech chart $6500 .{ }^{1314}$ The chart measures contrast sensitivity at five different spatial frequencies $(1.5,3,6,12$, and 18 cycles per degree). Each spatial frequency is presented in nine decreasing contrast steps and the patient's task is to identify the proper orientation of the bars (left, right, straight). The last correctly identified step is recorded as the result.

The literature ${ }^{13}$ shows that stray light caused by changes such as corneal oedema, corneal lesions, or lens opacities has a strong effect on the medium and high spatial frequencies. Therefore, the results were only analysed for 12 and 18 cycles per degree.

All measurements of visual acuity or contrast sensitivity were carried out with best correction for the testing distance.

The data were analysed by multiple regression.

\section{Results}

VISUAL ACUITY BY SNELLEN CHART

At the first month's visit, the best corrected visual acuity (BCVA), as measured with the Snellen chart, showed a clear reduction from a mean of $20 / 20$ to a mean of $20 / 24$, increasing gradually thereafter (Fig 2, curve 1). At the 6 month visit, the initial preoperative acuity had been regained $(p=0.7744)$. From the 6 month visit up to the 1 year visit, the VA remained stable. The three groups (I-III) did not display any difference in their sequence, but in the level of their acuity. The patients of group III (high myopes) had the lowest mean acuity value with $20 / 27$, compared with group II (moderate myopes) with 20/22, and group I (low myopes) with 20/18. Of the 46 eyes included in the study, 34 eyes $(74 \%)$ had improved on or attained their initial acuity within the year. Fifteen of the 34 eyes gained one line in VA. Of the patients who lost optimal corrected acuity, seven eyes lost 1 line compared with the preoperative level and five eyes lost 2 lines.

VISUAL ACUITY BY GLARE TEST (BERKELEY GLARE TEST)

The high contrast visual acuity (HCVA) measurements also decreased from 96.78 (SD 0.45) to 91.22 (SD 0.52) (VAR) at the first month's visit (Fig 2, curve 2). This corresponds with a VA reduction from $20 / 20$ to $20 / 25$. During the next 2 months, it increased again. The HCVA after 1 year reached 95.95 (SD 0.045) (VAR) and did not significantly differ from its original

Table 1 Overview of the visual acuity rating (VAR), the corresponding visual acuity decimal notation, and the Snellen visual acuity

\begin{tabular}{lll}
\hline VAR & Visus (decimal notation) & Visus (Snellen) \\
\hline $51-55$ & 0.125 & $20 / 160$ \\
$56-60$ & 0.16 & $20 / 125$ \\
$61-65$ & 0.2 & $20 / 100$ \\
$66-70$ & 0.25 & $20 / 80$ \\
$71-75$ & 0.32 & $20 / 63$ \\
$76-80$ & 0.4 & $20 / 50$ \\
$81-85$ & 0.5 & $20 / 40$ \\
$86-90$ & 0.625 & $20 / 32$ \\
$91-95$ & 0.8 & $20 / 25$ \\
$96-100$ & 1.0 & $20 / 20$ \\
$101-105$ & 1.25 & $20 / 16$ \\
$106-110$ & 1.6 & $20 / 12$ \\
$111-115$ & 2.0 & $20 / 10$ \\
\hline
\end{tabular}




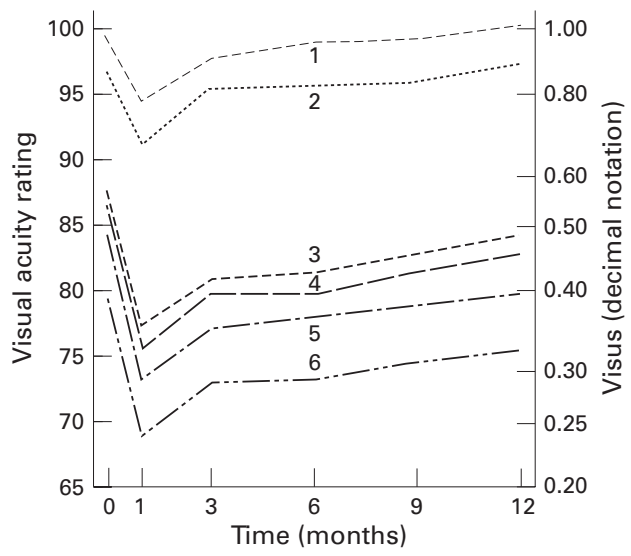

Figure 2 Sequence of visual acuity of optimally corrected Snellen visual acuity, high contrast visual acuity, and low contrast acuity with four different glare conditions: no glare; minimal glare; medium glare; maximum glare.

value $(\mathrm{p}=0.4834)$. There is a strong correlation between BCVA at distance and the HCVA measured with the Berkeley glare test $(r$ $=0.864)$.

All four curves of the results with the low contrast charts with maximum glare (Fig 2, curve 6 ), medium glare (Fig 2, curve 5), minimal glare (Fig 2, curve 4) and with no glare (Fig 2, curve 3 ) display a similar sequence. The variations between the curves are the absolute values, which increase with decreasing glare. Also noteworthy are the variations between the pre- and postoperative values after 1 year, which decrease with less glare. Under low contrast illumination at 1 year, VAR was measured as lower than the preoperative value for all test conditions.

Of significance is the low contrast visual acuity (LCVA) measured with maximum glare (curve 6). A large preoperative difference is seen between the HCVA of 96.78 (SD 0.45) and LCVA with maximum glare of 79.85 (SD 0.45 ) (Table 2). There was a sharp postoperative drop reaching its lowest point at 1 month (68.84 1 (SD 0.59) VAR). The acuity then improved up to the third month, with no further significant alteration thereafter. The low contrast visual acuity (LCVA) after 1 year remained significantly reduced in comparison with the initial values $(p=0.0011)$.

There is a high correlation between the curves. The correlation factor between maximum glare and medium glare is 0.964; between minimal glare and no glare, 0.982; and between maximum and no glare, 0.917 . If the patients are analysed by myopia grouping,
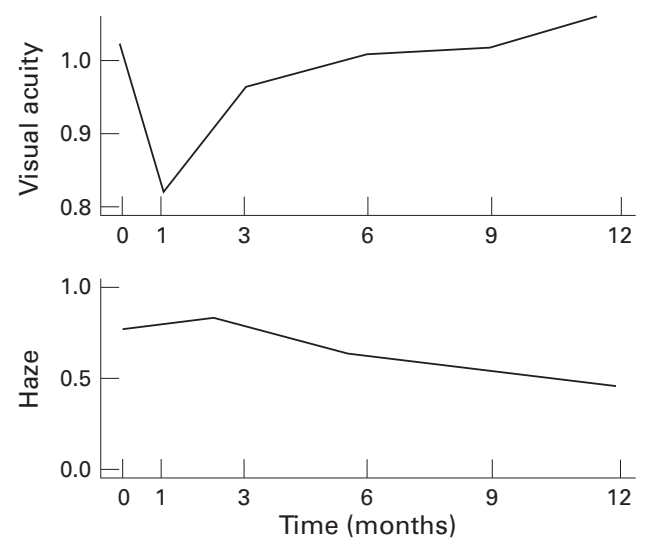

Figure 3 Follow up of Snellen visual acuity and haze over 1 year.

a clear difference in the VAR can be observed. Higher myopes showed a lower VAR. That result was independent of the manner in which acuity was measured (Snellen, HCVA, or LCVA with different glare sources). This difference existed preoperatively and maintained the same relation postoperatively.

VISUAL ACUITY - HAZE

The sequence of haze during the first 3 months (Fig 3) was contrary to that of VA. While the VA clearly recovers between the first and the third months, the degree of haze slightly increases during the same time period (from $0.778(0.0027)$ to $0.837(0.027))$. The haze continued to decrease, reaching a mean value of $0.429(0.035)$ at the 1 year checkup. Snellen BCVA had already reached its initial level after 6 month. If haze is analysed by the three refraction groups, the higher myopes had the highest degree of haze (up to 2.5), while for the other two groups the values did not exceed 1.5.

Comparing the Berkeley glare test results with that of haze grading points only to a weak correlation between the LCVA under the four different glare conditions and the haze. The correlation factor in the different cases fails to exceed -0.415 .

Even group III (high myopes) with the greatest degree of haze displayed only a weak correlation (LCVA maximum glare/haze $r=$ 0.395 and LCVA no glare/haze $r=0.333$ ).

\section{CONTRAST SENSITIVITY}

A contrast sensitivity plot, as measured with the Vistech 6500 chart, showed similarities to that of Snellen VA. Contrast sensitivity was

Table 2 Mean values (with standard deviation) of all patients: best corrected Snellen visual acuity, high contrast visual acuity, low contrast visual acuity with the four glare conditions (maximum, median, minimal, and no glare)

\begin{tabular}{lllllll}
\hline & 0 & 1 Month & 3 Months & 6 Months & 9 Months & 12 Months \\
\hline VCC & $1.02(0.014)$ & $0.82(0.015)$ & $0.96(0.018)$ & $1.01(0.018)$ & $0.99(0.017)$ & $1.02(0.018)$ \\
HCVA $^{*}$ & $96.78(0.45)$ & $91.22(0.52)$ & $95.47(0.48)$ & $95.74(0.45)$ & $95.14(0.48)$ & $95.95(0.45)$ \\
LCVA max G $^{\star}$ & $79.85(0.45)$ & $68.84(0.59)$ & $72.98(0.51)$ & $73.15(0.55)$ & $73.76(0.51)$ & $74.20(0.53)$ \\
LCVA mid G $^{\star}$ & $84.30(0.47)$ & $73.25(0.61)$ & $77.09(0.51)$ & $77.89(0.51)$ & $78.16(0.50)$ & $78.50(0.51)$ \\
LCVA min G $^{\star}$ & $86.67(0.47)$ & $75.66(0.56)$ & $79.58(0.53)$ & $79.70(0.50)$ & $80.70(0.51)$ & $81.30(0.48)$ \\
& $87.63(0.49)$ & $77.32(0.51)$ & $80.89(0.51)$ & $81.41(0.49)$ & $82.03(0.49)$ & $83.20(0.47)$ \\
\hline
\end{tabular}

To facilitate interpretation of the results, visual acuity is mentioned in decimal notation. $0=$ preoperative control; VCC $=$ Snellen visual acuity with correction in decimal notation; ${ }^{\star} \mathrm{VAR}=$ visual acuity rating; HCVA $=$ high contrast visual acuity; LCVA $=$ low contrast visual acuity; max $\mathrm{G}=$ maximum glare $\left(3000 \mathrm{~cd} / \mathrm{m}^{2}\right) ;$ mid $\mathrm{G}=$ medium glare $\left(800 \mathrm{~cd} / \mathrm{m}^{2}\right) ; \operatorname{min~} \mathrm{G}=$ minimal glare $(300$ $\left.\mathrm{cd} / \mathrm{m}^{2}\right) ;$ no $\mathrm{G}=$ no glare (only front illumination). 


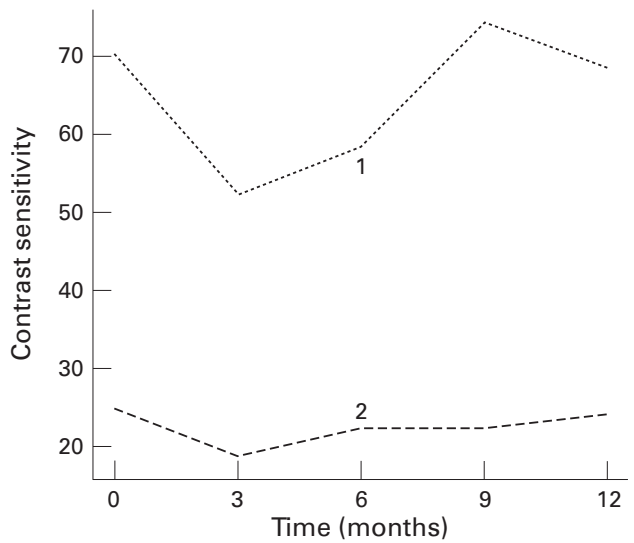

Figure 4 Diagram showing contrast sensitivity (Vistech chart), at the spatial frequencies of: 12 cycles/degree (1) and 18 cycles/degree (2).

found first to decrease at the 1 and 3 month postoperative checks, later increasing up to the 1 year visit. One year postoperatively, there was no significant difference from the preoperative value $(\mathrm{p}=0.8342)$. The curves of both spatial frequencies show their lowest points at the 3 month visit with 52.48 (2.75) (12 cycles/ degree) and 18.65 (1.15) (18 cycles/degree) (Fig 4). The curve of the spatial frequency at 12 cycles/degree showed an increase during the following 6 months exceeding the initial value $(\mathrm{p}=0.8342)$. The curve of the 18 cycles/ degree frequency showed a steady increase up to the 1 year visit, slightly exceeding its initial value $(26.71(1.36))$. There was also no significant difference between the 1 year visit and its initial value $(\mathrm{p}=0.7936)$. Contrast sensitivity showed no correlation with haze $(r=0.332)$.

REGRESSION RATE

The mean deviation of the achieved refraction from the aimed refraction 6 months postoperatively was $0.06 \mathrm{D}$ (SD $0.193 \mathrm{D})$. One year postoperatively, the mean deviation increased to $-0.559 \mathrm{D}(\mathrm{SD} 0.183 \mathrm{D}$ ) and differed significantly $(p=0.004)$ from the aimed refraction.

If the aimed refractions and the 1 year postoperative refractions are analysed by myopia grouping, a clear difference can be observed. Group I with low myopia shows a difference from the aimed refraction of $0.284(0.179) \mathrm{D}$, which is not significant $(\mathrm{p}=0.142)$. Group II (moderate myopia) shows a mean difference of $-0.704(0.217) \mathrm{D}$, which is significant $(\mathrm{p}=$ 0.005 ), and group III (high myopia) shows a mean difference of $-0.586(0.593) \mathrm{D}$, which is not significant $(\mathrm{p}=0.348)$. All groups present a differential increase between the aimed and achieved refraction from the 6 to the 12 month visits.

Interestingly, mean Snellen BCVA returned to its initial value after 6 months, while mean HCVA recovered only after 1 year. This time discrepancy could be the result of different measurement methods. Final BCVA was recorded when four out of five letters were correctly identified, while each letter correctly identified with Berkeley glare test added up to the final VAR.

\section{Discussion}

A more accurate measure of VA can be achieved with the Bailey-Lovie chart, as used by the Berkeley glare test. With this chart, the spacing between the letters is related to letter size and the steps between lines are equivalent. A disadvantage with normal VA charts is that spacing between letters increased while letter size decreases.

In all, $74 \%$ of the treated eyes reached the initial VA or gained VA after 1 year, $26 \%$ did not reach their initial VA even after 1 year.

A similar outcome was described by Gartry et $a l^{2}$ in a study of preoperative myopia between -1.5 and $-17.5 \mathrm{D}$ (spherical equivalent). After modifying and optimising the surgery, no patient lost more than one line of BCVA in the described set up. ${ }^{21}$

Some studies also considered the contrast sensitivity. ${ }^{22-25}$ Seiler and Wollensak ${ }^{26}$ showed in a study of 26 eyes/patients with a preoperative myopia between -1.4 and $-9.25 \mathrm{D}$ (spherical equivalent) operated on with an Eximed UV299 (Summit Technology), a similar development of the visual acuity. They also examined the VA with glare (Humphrey Mod 570). With that measurement arrangement, the patients achieved a preoperative VA of $20 / 25$ with activated glare source. During the first month, the average VA dropped to $20 / 40$ and then increased to $20 / 36$ at the 3 month period. Even after 1 year, when VA measured 20/30 with activated glare source, it remained at a lower level in comparison with its preoperative level.

Even though all groups displayed a postoperative regression of refraction, it did not affect the results, since all measurements were performed with optimal correction for the appropriate test distances.

Our patients also exhibited a VA between 20/50 (maximum glare) and 20/32 (no glare) when measured with the low contrast chart $(10 \%)$. There was a significant deterioration in VA during the first month (20/80 with maximum glare and 20/50 with no glare). At the 3 month visit, the acuity with maximum glare had increased to $20 / 60$ and with no glare to $20 / 40$. At the 1 year visit, the VA was on average one line lower than the initial VA. The difference between low contrast and high contrast VA (as measured with Snellen charts and the $96 \%$ contrast charts of the Berkeley glare test) 1 year postoperatively shows clearly that visual function cannot be judged accurately and completely by conventional visual acuity charts.

The study shows clearly that the Vistech chart does not yield sufficiently sensitive information with respect to the reduction in the quality of vision after PRK. This was also supported by the result of the multicentre study by Sher et $a l,{ }^{23}$ who also used the Vistech chart and found no significant difference between the values before and 3 months after treatment. One reason for this may be the nature of the test-a selection of bars from only three available directions implies that the element of chance in choosing the correct direction is higher than selecting one of 26 letters. The 
Vistech chart is not sensitive enough to differentiate subtle changes.

Our results also support Nadler and $\mathrm{Miller}^{27}$ in their conclusion that a deterioration in the visual function cannot be analysed correctly using only a low contrast chart; a glare source must be added to facilitate correct evaluation of the reduction.

What is the cause of the contrast sensitivity loss? The simplest answer would be the haze. A thorough analysis shows that it can be, at most, an additional factor. This study has demonstrated, as others before, ${ }^{2426}$ that haze increases between the first and third month after PRK. On the other hand, VA already increases between the first and third month, regardless of whether it is measured with $96 \%$ or $10 \%$ contrast charts, with or without glare. This statement is supported by the lack of correlation between haze and Snellen VA, ${ }^{2}$ and the weak correlation between haze and low contrast visual acuity with or without glare.

Morphological changes in the cornea, such as haze, can cause incoming light to be back scattered, making the haze visible to the observer. Part of the incoming light is also scattered to the retina (forward scatter). This light causes a deterioration of the retinal image, a reduction of the contrast sensitivity, and a possible increase in glare sensitivity. The Berkeley glare test measured the contrast sensitivity in view of the forward light scatter. Our study concluded that the increase in glare sensitivity can only partially be attributed to the haze; other factors also contribute to the phenomenon.

Other possible causes discussed include spherical aberration of the abnormal corneal topography, ${ }^{28}$ decentration, and the effect of the optical junction between the ablated and the non-treated corneal area on large pupils. This last point gains in importance for small ablated zones. The diameter of the ablation zone used in our study was between 5 and 7 $\mathrm{mm}$, with a transition zone of 1-2 $\mathrm{mm}$ (TTZ). It will be interesting to see if larger ablation zones have a beneficial effect on the outcome, particularly for the high myopes of group III. This will be the focus of our next investigation.

\section{Conclusion}

The study clearly demonstrates that, even 1 year after PRK with the MEL 60 excimer laser, a reduced contrast sensitivity can be measured with the Berkeley glare test, with or without glare. This deterioration of vision, which most patients do not notice, needs to be discussed with the patient and has to be taken into consideration when counselling for excimer surgery, since it may be of importance in certain circumstances (professional, driving, later onset of other pathological conditions). Excimer laser technology and treatment need to take into account improvements that will reduce glare sensitivity. The future will show if new technology and a broader flattening area of 6-7 $\mathrm{mm}$ will positively affect the outcome.
We thank Dr Mark Bullimore for supplying the Berkeley test charts and Drs Philipp Hendrikson and William Burnham for help in preparing this manuscript.

1 Seiler T, Kahle G, Kriegerowski M. Excimer laser (193 nm) myopic keratomileusis in sighted and blind human eyes. Refract Corneal Surg 1990;6:165-73.

2 Gartry DS, Kerr Muir MG, Marshall J. Photorefractive keratectomy with an argon fluoride excimer laser: a clinical study. Refract Corneal Surg 1991;7:420-35.

3 Lohmann CP, Timberlake GT, Fitzke FW, Gartry DS, Kerr Muir MK, Marshall J. Corneal light scattering after excimer laser photorefractive keratectomy: the objective measurements of haze. Refract Corneal Surg 1992;8:11421.

4 Lohmann CP, Gartry DS, Kerr Muir M, Timberlake GT, Fitzke FW, Marshall J. 'Haze' in photorefractive keratectomy: its origins and consequences. Laser Light Ophthalmol 1991;4:15-34.

5 van den Berg TJ. On the relation between glare and stray light. Doc Ophthalmol 1991;78:177-81.

6 Holladay LL. The fundamentals of glare and visibility. $f \mathrm{Opt}$ Soc Am 1962;12:271.

7 LeClaire J, Nadler MP, Weiss S, Miller D. A new glare tester for clinical testing. Results comparing normal subjects and variously corrected aphakic patients. Arch Ophthalmol 1982;100:153-8.

8 Smith PW , Pratzer KA, Webster N, Fenton J, Bonham RD. A clinical comparison of two methods of glare testing. Ophthalmic Surg 1987;18:680-2.

9 Hirsch RP. Evaluation of the brightness acuity tester (letter). f Cataract Refract Surg 1987;13:341-2.

10 Elliott DB, Hurst MA, Weatherill J. Comparing clinical tests of visual loss in cataract patients using a quantification of forward light scatter. Eye 1991;5:601-6.

11 Regan D. The Charles F. Prentice Award Lecture 1990: specific tests and specific blindnesses: keys, locks, and parallel processing. Optom Vis Sci 1991;68:489-512.

12 Pelli DG, Robson JG, Wilkins AJ. The design of a new letter chart for measuring contrast sensitivity. Clin Vis Sci 1988;2:187-99.

13 Ginsburg AP. The evaluation of contact lenses and refractive surgery using contrast sensitivity. In: Contact lenses. New York: Grune and Stratton, 1987:56.1-56.17.

14 Reeves BC, Wood JM, Hill AR. Vistech VCTS 6500 Charts - within- and between-session reliability. Optom Vision Sci 1991;9:728-37.

15 Bailey IL, Bullimore MA. A new test for the evaluation of disability glare. Optom Vision Sci 1991;68:911-7.

16 American Academy of Ophthalmology. Contrast sensitivity and glare testing in the evaluation of anterior segment disease. Ophthalmology 1990;97:1233-7.

17 Elliott DB, Bullimore MA. Assessing the reliability, discriminative ability, and validity of disability glare tests. Invest Ophthalmol Vis Sci 1993;34:108-19.

18 Zürcher M, Schipper I, Schupfer G, Senn P. The excimer laser. II Initial experiences with the excimer laser in Lucerne. Klin Monatsbl Augenheilkd 1992;200:338-42.

19 Bailey IL, Lovie JE. New design principles for visual acuity letter charts. Am f Optom Physiol Optics 1976;53:740-5.

20 Fantes FE, Hanna KD, Waring GO, Pouliquen Y, Thompson KP, Savoldelli M. Wound healing after excimer laser keratomileusis (photorefractive keratectomy) in monkeys (see comments). Arch Ophthalmol 1990;108:665-75.

21 Schipper I, Senn P, Lechner A. Tapered transition zone and surface smoothing ameliorate the results of excimer-laser photorefractive keratectomy for myopia. Ger $\mathcal{F}$ Ophthalmol 1995;4:368-74.

22 Seiler T, Wollensak J. Results of a prospective evaluation of photorefractive keratectomy at 1 year after surgery. Ger $\mathcal{F}$ Ophthalmol 1993;2:135-42.

23 Sher NA, Chen V, Bowers RA, Frantz JM, Brown DC, Eiferman R, et al. The use of the 193-nm excimer laser for myopic photorefractive keratectomy in sighted eyes. A multicenter study (see comments). Arch Ophthalmol 1991;109:1525-30.

24 Lohmann CP, Gartry DS, Kerr Muir MK, Timberlake G, Fitzke F, Marshall J. Corneal opacity after photorefractive keratectomy with an excimer laser. Cause, objective measurement and functional consequences. Ophthalmologe measurement and

25 Ficker LA, Bates AK, Steele ADMcG, Lyons CJ, Milliken $\mathrm{AB}$, Astin $\mathrm{C}$, et al. Excimer laser photorefractive keratectomy for myopia: 12 month follow-up. Eye 1993;7:617-24.

26 Seiler T, Wollensak J. Myopic photorefractive keratectomy with the excimer laser. One-year follow-up. Ophthalmology 1991;98:1156-63.

27 Nadler MP, Miller D, Nadler DJ. Glare and contrast sensitivity in cataracts and pseudophakia. In: Glare and contrast sensitivity for clinicians. Berlin: Springer-Verlag 1990:53-65.

28 Seiler T, Reckmann W, Maloney RK. Effective spherical aberration of the cornea as a quantitative descriptor in corneal topography. $\mathcal{F}$ Cataract Refract Surg 1993;19:155-65. 